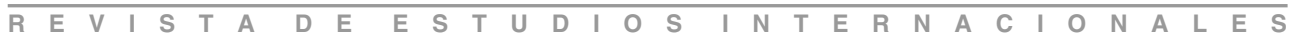

\title{
El foro internacional de la ASEAN y la República Popular China
}

\author{
Martín Pérez Le-Fort
}

La relación de competencia estratégica sinonorteamericana es un elemento sustantivo de la seguridad del este de Asia. Pese a que últimamente han surgido expectativas de mejorar la relación bilateral, no se han alterado de manera sustancial las opciones estratégicas de ninguno de ellos, lo que se debe en parte a la percepción negativa china respecto de la fuerte influencia norteamericana en la región. En el sudeste asiático, las disputas en el mar del Sur de China son un espacio sutil de dicha competencia en que confluyen, por una parte, la necesidad china de garantizar su seguridad energética, sus pretensiones de soberanía sobre gran parte de dicho mar y, por la otra, la perspectiva norteamericana, que pone un marcado acento en la contención de China y su interés por ampliar su presencia en el plano militar, algo debilitado en la post Guerra Fría. Esto ha llevado a China a revalorizar los esquemas de seguridad multilateral regional, y perseguir un mayor grado de participación, coincidente con el objetivo de construir un mundo multipolar. La activa participación china en el foro Regional de la ASEAN obedece a que es una instancia oportuna para garantizar la estabilidad de la situación estratégica regional, necesaria para facilitar la integración económica y abrir espacios de negociación, pero sin discutir directamente temas de soberanía. Dicha instancia se hace relevante principalmente por su flexibilidad y por el bajo grado de formalización.

\section{INTRODUCCIÓN}

na organización multilateral que ha despertado gran interés en el ámbito de la seguridad dentro del Asia-Pacífico es el Foro Regional de la ASEAN. Dicha organización, surgida en el sudeste asiático bajo el amparo de la
Asociación de Naciones del Sudeste, asiático (ASEAN), se ha constituido como la principal instancia de diálogo de seguridad en la Cuenca del Pacífico.

Las particulares características del sudeste asiático han generado las condiciones para la evolución de dicha organización. A pesar de la visión ampliamente 
aceptada del sudeste asiático como una región política construida en términos conceptuales con posterioridad a la Segunda Guerra Mundial, no se puede obviar el hecho de las intensas relaciones entre los Estados precoloniales, cientos de años antes del impacto del fenómeno colonial en la región, principalmente en el Sudeste asiático continental. Sin embargo, las condiciones geográficas restringieron la interacción entre los Estados continentales e insulares, estableciéndose un factor inicial de heterogeneidad. Por otra parte, la penetración cultural de China e India, aunque no profunda, aumentó la falta de coherencia cultural y de valores de la zona ${ }^{1}$.

\section{Las condiciones geográficas limitaron la interacción entre Estados continentales e insulares.}

Durante la ocupación colonial, la principal característica de los países de la región fue su orientación de los Estados hacia la metrópoli, más que hacia los Estados vecinos. La rápida destrucción de dicho orden por parte de Japón, en 1942, reintrodujo las relaciones entre los países de la región y sentó las bases para el surgimiento de un nacionalismo que impidió el restablecimiento del colonialismo sobre bases permanentes. La consolidación de los Estados nacionales tuvo un elemento común, el cual fue la presencia e influencia de las superpotencias en bus- ca de la hegemonía, que estableció un nuevo factor de división. El conflicto ideológico fue la principal forma de división del Sudeste asiático, es decir, la que se dio entre los países comunistas de Indochina y los países bajo influencia occidental. Cabe notar que el nacionalismo siempre estuvo presente, aunque fuera de forma latente, en los conflictos que parecían tener un carácter meramente ideológico.

Pese a la gran heterogeneidad de la región, hubo esfuerzos por estrechar los lazos entre los países no comunistas. Un esfuerzo que logró fructificar fue la ASEAN, creada en 1967 y cuyo propósito fue promover las relaciones económicas, sociales, y culturales entre sus miembros. Inicialmente, ellos fueron Indonesia, Malasia, Singapur, Tailandia y Filipinas y, luego Brunei que se incorporó en 1984. Durante la década de los noventa se integraron Myanmar y los países de Indochina (Vietnam, Laos y Camboya). Aunque jamás se planteó formalmente, el propósito político subyacente de la ASEAN fue crear un entorno en el cual la sobrevivencia de cada Estado sería asegurada a través del fomento de la estabilidad regional y la limitación de la competencia entre ellos ${ }^{2}$.

\section{LA ASEAN y LA SEGURIDAD}

El tema de la seguridad regional ha sido recurrente, debido a que la zona fue

${ }^{1}$ Dibb, Paul, "Toward a New Balance of Power in Asia", Adelphi Paper 295, mayo de 1995, p. 11.

${ }^{2}$ Palmer, Ronald D. y Reckford, Thomas J., Building ASEAN. 20 Years of Southeast Asian Cooperation, The Center for Strategic and International Studies, Washington, D.C, 1987, p. 5. 
un importante terreno de lucha hegemónica por parte de las superpotencias. La Guerra Fría, reflejada en el conflicto de Vietnam, delineó la primera fase del desarrollo de la ASEAN en el ámbito de la seguridad, fundamentalmente en términos de una hegemonía norteamericana y la amenaza de la insurgencia comunista, que tuvo como un hito importante la caída de Vietnam del Sur en $1975^{3}$.

\section{La zona fue importante terreno de lucha hegemónica por las superpotencias.}

Las metas fundacionales de la ASEAN eran de suma importancia porque las entonces prevalecientes insurgencias indígenas (principalmente comunistas), amenazaban socavar no sólo los Estados mismos, sino también la estabilidad regional, con la posible consecuencia de involucrar a poderes externos no deseados. Esta estrategia fue expresada en 1971 con la Declaración sobre la Zona de Paz, Libertad y Neutralidad (ZOPFAN) con la idea de paz a través de la neutralidad. El ideal de llegar a la ZOPFAN se vio reflejado en el propósito de crear una zona libre de armas nucleares en el sudeste asiático (SEANWFZ) planteado en la reunión de Ministros de ASEAN en 1984, para restringir las opciones nucleares de las potencias globales y que se constituía en un paso hacia este ideal. El Tratado de Amistad y Cooperación de 1976 dio las bases legales para el ZOPFAN y junto con la Declaración de Concordia de la ASEAN, del mismo año, proveyeron los instrumentos para manejar el orden regional. El Tratado de Amistad incluía mecanismos para la solución pacífica de controversias y la renuncia del uso de la fuerza contra otros miembros. La concordia sumaba la idea de resiliencia (autoseguridad) nacional y de la ASEAN.

La unificación de Vietnam en 1975 y la posterior invasión de Camboya por ese país, en 1978, que reflejaba la disputa mayor entre los intereses chinos y de la Unión Soviética, dieron lugar a una segunda etapa en la evolución institucional de ASEAN, además de un mayor acercamiento con la República Popular China (China), ante la amenaza vietnamita. La consecuencia más evidente de este nuevo contexto fue el establecimiento del secretariado de la ASEAN en Indonesia, que reflejaba el fuerte acento en el sudeste asiático marítimo, con dicho país como primus inter pares dentro de dicha organización ${ }^{4}$.

La crisis de Camboya trajo tensión a la región e impidió implementar la ZOPFAN. El propósito de hacer del sudeste asiático una zona libre de armas nucleares fue una medida para llegar al ZOPFAN. Estados Unidos se oponía a este propósito, ya que poseía bases en la región. La imposibilidad de implementar la ZOPFAN no fue obstáculo para que aumentara el diálogo, se aplicara una serie de medidas y se realizaran diversas reuniones para incrementar la cooperación

${ }^{3}$ Ganesan, N., “ASEAN's Relations with Major External Powers”, Contemporary Southeast Asia, Vol. 22, $N^{\circ}$ 2, agosto de 2000, pp. 260-263.

${ }^{4}$ Ganesan, N, Ibidem, p. 263. 
y evitar el conflicto entre sus miembros. Este diálogo le dio el sentido de identidad a dicha organización, al desarrollarse intereses y valores comunes, además de los intereses nacionales. A pesar de mantener conflictos territoriales entre ellos, los países miembros no contemplaron el uso de la fuerza para resolver sus problemas.

\section{Pese a conflictos territoriales entre ellos, los países de la ASEAN no contemplaron el uso de la fuerza para resolverlos.}

El conflicto de Camboya fue la primera amenaza a la paz de la región. Sin embargo, fue claro que ninguno de los países que conformaban la ASEAN tenían la intención de usar unilateralmente la fuerza para revertir la situación y optaron por aumentar la cooperación con actores externos en el uso de una variedad de instrumentos políticos, económicos, diplomáticos y militares. La solución al problema de Camboya en 1991, mediante el acuerdo de París, adoptó la fórmula de la ASEAN que era: total retirada de las tropas vietnamitas de Camboya; autodeterminación para el pueblo camboyano y, finalmente, una Camboya independiente y neutral.

Sin embargo, la parcial solución del problema de Camboya se debió más a los profundos cambios a nivel global, principalmente a la caída de la Unión Soviética (principal socio militar de Vietnam en ese período), y el consiguiente fin de la bipolaridad. Cabe observar que entre los países miembros no había una visión similar respecto de China y del papel de Vietnam, país que siempre se mostró intransigente ante la postura de ASEAN.

La Guerra Fría provocaba la rápida internacionalización de los conflictos regionales y la consiguiente polarización entre la Indochina comunista y la ASEAN no-comunista. Por otra parte, la insurgencia de origen comunista era un factor común entre los miembros, lo que ayudaba aún más a la polarización. Cabe notar que dentro de la ASEAN se mantenían numerosas disputas limítrofes.

Dentro de estas disputas limítrofes, uno de los conflictos que ha generado gran interés en los países de la región es el conflicto del archipiélago de las Spratly, en el Mar del Sur de China. El archipiélago es un conjunto de más de 230 islotes, arrecifes y bancos de arena que cubren un área de 250.000 kilómetros cuadrados y que ha llegado a ser un obstáculo para la creación de estructuras de seguridad multilaterales en la región. China, Vietnam, Taiwan, Filipinas, Malasia y Brunei reclaman la totalidad o parte de las islas Spratlys y el área marítima circundante, cuyo lecho marino contendría petróleo y gas. Además, las islas tienen gran significación para la defensa de rutas marítimas, la interdicción y vigilancia para potencias mayores y menores. Además, las disputas afectan los intereses económicos y de navegación de Japón y Estados Unidos.

Por otra parte, a pesar de la tendencia hacia el incremento de la cooperación, la ASEAN no está exenta de conflictos ${ }^{5}$.

${ }^{5}$ Acharya, Amitav, "A New Regional Order In South-East Asia: ASEAN in the Post-Cold War Era”, Adelphi, Paper 279, Agosto 1993, pp. 7-12 y pp. 30-33. 
Ellos incluyen el conflicto sobre las Spratlys, que involucra a cuatro miembros de la ASEAN (Filipinas, Malasia, Brunei y Vietnam); las disputas entre Malasia y Singapur sobre la isla de Pedra Branca, cerca de las costas de Johor, entre Malasia e Indonesia, respecto de las islas Ligitan y Sipadan, en el Mar de Sulawesi, entre Malasia y Brunei respecto del territorio de Limbang, en Sarawak, entre Malasia y Filipinas, respecto de Sabah, etc.

Además de conflictos interestatales, hay otros aspectos que afectan la seguridad de la región, entre los que pueden destacarse el terrorismo, la creciente presencia china en Myanmar y los problemas de separatismo que enfrenta Indonesia y que han dado pie a graves crisis, como lo fue el caso de Timor Oriental, además de otros conflictos internos no resueltos a lo largo de toda la región.

El sudeste asiático es una región en proceso de transición estratégica debido a la disminución de la presencia militar directa de las superpotencias. Como ya hemos visto, dicha región ha enfrentado la permanente influencia de actores extrarregionales y la existencia de variados conflictos limítrofes e internos aún no resueltos, lo que ha tenido un fuerte impacto tanto en los sistemas de seguridad multilateral regionales como en las políticas de defensa y seguridad de cada país en particular.

En esta región se han desarrollado sistemas de seguridad multilateral regional que responden principalmente a una percepción disímil, y a veces contradictoria respecto de la acción de potencias regionales (China, Estados Unidos, India, la
Federación de Rusia, Japón, etc). Por otra parte, las políticas de seguridad y defensa de los países del sudeste asiático responden a ese mismo tipo de percepción, lo que sumado a los variados conflictos limítrofes puede inducir a carreras armamentistas en la región a partir de percepciones erradas de la seguridad.

\section{El sudeste asiático es una región en proceso de transición estratégica.}

El sudeste asiático posee una arquitectura de seguridad multilateral en la cual coexisten estructuras de seguridad, colectiva, comprehensiva y cooperativa. Debido a que la estructura de seguridad multilateral regional apunta principalmente a las amenazas extrarregionales, no se ha logrado una solución eficaz de los conflictos territoriales intrarregionales, que se mantienen latentes. Esta particular arquitectura de seguridad, que supuestamente busca limitar la intromisión de potencias extrarregionales, no logra una forma coherente de enfrentar esta clase de amenazas. Esto ha llevado a establecer principalmente formas de cooperación de carácter informal en el ámbito de la seguridad, que incorporen el mayor número de partes involucradas y que se aboquen inicialmente a temas no contenciosos de interés común, tales como el manejo de problemas medioambientales, la piratería, la gestión de los recursos marinos, entre otros, respecto de los cuales se han registrado algunos avances.

Un aspecto característico de ASEAN en la post-Guerra Fría, ha sido el desarro- 
llo de métodos pacíficos de solución de conflictos, siendo casos emblemáticos los de Camboya y posteriormente el de las Spratlys. La idea de este Foro parte en 1990, con los discursos del canciller australiano Gareth Evans respecto de la idea de una seguridad común según el modelo de la CSCE (Conferencia sobre Seguridad y Cooperación en Europa). En un principio, la idea parecía ser generar estructuras similares en Asia. Al comienzo esto fue visto con suspicacia por los países miembros, que no veían aplicable directamente la fórmula de construcción de medidas de confianza mutua y la de construcción de seguridad utilizadas en Europa, por lo cual el interés se centró en la creación de un foro para la seguridad en el Asia-Pacífico. Para ello, se aprovecharon las conferencias postministeriales de la ASEAN (ASEAN-PMC) iniciadas en 1978.

\section{Se miraba con suspicacia la idea de seguridad común al estilo europeo.}

Esta iniciativa recibió el apoyo de Japón y una fría respuesta de Estados Unidos en 1991. En la ASEAN-PMC de 1991, se anunció que podrían darse discusiones de seguridad en su seno. Inicialmente fue rechazada por Estados Unidos la idea, pero la llegada de la administración Clinton trajo consigo un cambio de su postura, ya que le dio todo su apoyo. En julio de 1993, la ASEAN anunció el establecimiento del Foro Regional de la ASEAN (ARF), cuya reunión de trabajo tuvo lugar en Bangkok en mayo de 1994 y de cancilleres en julio de 1994.

En el contexto regional, el Foro Regional de la ASEAN ha servido para la búsqueda de solución a diversos conflictos, dentro de los que podemos destacar el problema de Camboya, la disputa por las islas Spratly -que involucra a China y a varios países del sudeste asiático- la situación política de Myanmar o la lucha contra el terrorismo. Entre sus objetivos fundamentales están: fomentar el diálogo constructivo y la consulta sobre problemas políticos y de seguridad relacionados con intereses y preocupaciones comunes; emprender esfuerzos para la construcción de confianza y diplomacia preventiva en la región; y trabajar hacia el fortalecimiento y mejora de la cooperación en la seguridad política dentro de la región como un medio de asegurar la paz y estabilidad $^{6}$. Para llevar a cabo ese proceso, el ARF elaboró un plan en tres etapas que incluye: la promoción de medidas de construcción de confianza; el desarrollo de mecanismos de diplomacia preventiva y; el desarrollo de mecanismos de solución de conflictos. Este organismo, junto con otras instancias de diálogo informal (también conocidas como de "track two"), tales como los CSCAP's (National Councils for Security Cooperation in the AsiaPacific) han cumplido una función relevante e innovadora en la seguridad regional ${ }^{7}$.

${ }^{6}$ Caballero-Anthony, Mely, "Partnership for Peace in Asia: ASEAN, ARF, and the United Nations", Contemporary Southeast Asia, Vol. 24, № 3, diciembre 2002, p. 535.

${ }^{7}$ Simon, Sheldon W., "Evaluating Track II approaches to security diplomacy in the Asia-Pacific: the CSCAP experience", The Pacific Review, Vol. 15, N 2, 2002, pp. 167-200. 
La relaciones de seguridad en la región se han visto comprometidas en gran medida por las disputas limítrofes en el Mar del Sur de China, que involucran a China y varios países miembros de la ASEAN, generando una fuerte percepción de amenaza respecto de China. El rol del ARF está generando un gran interés debido a que ha logrado incorporar en un proceso de diálogo franco y abierto a todos los involucrados en esas disputas, reafirmando un papel creciente como base de una arquitectura de seguridad, no sólo del sudeste asiático, sino que potencialmente del Asia-Pacífico en su conjunto. La idea de una profundización del papel del ARF tiene el respaldo incluso de China, que está procurando ampliar el papel de la instancia en función de su competencia estratégica con Estados Unidos, rescatando recientemente la idea de establecer, bajo el paraguas del ARF, un foro de seguridad que incorpore a personal militar de los países del Asia-Pacífico ${ }^{8}$.

El ARF se compone de 23 Estados, entre los que se cuentan los de ASEAN10 (Brunei Darussalam, Camboya, Indonesia, Laos, Malasia, Myanmar, Filipinas, Singapur, Tailandia y Vietnam) y los "socios de diálogo" de la ASEAN (Australia, Canadá, China, la Unión Europea, India, Japón, Corea del Sur, Nueva Zelandia, Rusia y Estados Unidos). La importancia del Foro Regional de la ASEAN como instancia especializada en el ámbito de la se- guridad radica en su carácter inclusivo, aunque el fuerte grado de flexibilidad institucional que posee se visualiza como su fortaleza o debilidad, según el enfoque teórico de que se trate.

\section{El foro regional de la ASEAN es modelo de regionalismo $\mathrm{y}$ cooperación en el Tercer Mundo.}

Esta organización es considerada como un modelo de regionalismo y cooperación en el Tercer Mundo. La ASEAN ha dado a los países miembros mayores niveles de reputación y audiencia internacional de los que podrían haber alcanzado por sí solos. La incorporación de los países de Indochina y Myanmar a la ASEAN en la década de los noventa, partiendo por Vietnam en 1995, ha generado un nuevo entorno muy promisorio respecto del incremento de la cooperación regional.

El tema del terrorismo ha dado pie a respuestas diversas en el sudeste asiático, debido a que algunos de ellos son países musulmanes (Indonesia y Malasia), lo que dificulta el manejo del tema debido a limitaciones de política interna9 ${ }^{9}$ Este aspecto se ha reflejado en la dificultad de la ASEAN de implementar política efectivas contra las redes terroristas. El atentado de Bali, Indonesia, de octubre de 2002 demostró la vulnerabilidad del gobierno de ese país ${ }^{10}$. Esto ha derivado en

${ }^{8}$ ABC News, 19 de junio de 2003.

${ }^{9}$ East Asian Strategic Review, 2002, Tha National Institute for Defense Studies, Tokio: The Japan Times Ltd., 2003; p. 19.

${ }^{10}$ East Asian Strategic Review, 2003. The National Institute for Defense Studies, Tokio: The Japan Times Ltd., 2003; p. 45. 
esfuerzos para fortalecer el ARF, como lo refleja la iniciativa de establecer una secretaría, centros de investigación de seguridad regional y reducir los riesgos, entre otras medidas importantes ${ }^{11}$.

\section{Se duda de la capacidad de las instituciones regionales de manejar conflictos y preservar la paz.}

\section{LA SEGURIDAD EN EL SUDESTE ASIÁTICO Y EL DEBATE TEÓRICO}

Desde el punto de vista de las relaciones internacionales, los enfoques teóricos principales que analizan la seguridad de la región y el papel del ARF en ella, derivan principalmente del liberalismo o neoliberalismo, del neorrealismo y del constructivismo.

El liberalismo enfatiza el papel de los actores estatales y no estatales y ve a la política internacional en términos de un juego de suma positiva en contraposición al modelo de suma cero propio del realismo. El modelo liberal presta atención al efecto mitigante de la interdependencia, las instituciones multilaterales y los regímenes internacionales sobre la anarquía internacional. Las principales características de este modelo son los efectos pacificadores del comercio, los efectos pacificadores del gobierno republicano, la importancia que las reglas e instituciones tienen para afectar las relaciones entre los países; $y$, finalmente, el efecto transformador de los contactos transnacionales y coaliciones en las actitudes nacionales y las definiciones de intereses ${ }^{12}$.

Para el "institucionalismo" neoliberal, como variante del liberalismo, los efectos pacificadores del multilateralismo conciben el desarrollo de éstos más en términos de procesos de socialización y construcción de consensos que en términos de estructuras legalistas formales, y perciben el ARF como una herramienta útil para manejar las incertidumbres estratégicas en la post Guerra Fría ${ }^{13}$. Además de la pertinencia del enfoque "realista", se ha ido incorporando el enfoque "neoliberal" al análisis de la seguridad, a través de la creación de regímenes e instituciones para la solución pacífica de controversias $^{14}$. Esto ha dado paso al estudio de la posible aplicación de conceptos tales como seguridad comprehensiva, común y cooperativa en la región ${ }^{15}$. Asimismo se ha puesto énfasis en problemas teóricos respecto de la teoría de seguridad, buscando determinar si la región es

${ }^{11}$ Ibid., pp. 223-224.

${ }^{12}$ Yong, Deng, "The Chinese Conception of National Interests in International Relations", The China Quarterly, junio 1998, No 154, pp. 308-329.

${ }_{13}$ Acharya, Amitav, "Realism, Institutionalism, and the Asian Economic Crisis", Contemporary Southeast Asia, Vol. 21, No 1, April 1999, p. 5

${ }^{14}$ Simon, Sheldon W., "Realism and Neoliberalism: International Relations Theory and Southeast Asian Security", The Pacific Review, Vol. 8, № 1, 1995, pp. 5-24

${ }^{15}$ Dewitt, David, "Common, Comprehensive and Cooperative Security", The Pacific Review, Vol. 7 , $\mathrm{N}^{\circ} 1,1994$, pp. 1-15. 
un complejo, una comunidad o un régimen de seguridad ${ }^{16}$. Dentro de estos enfoques también ha ganado fuerza la aplicabilidad del concepto de "vacío de poder", como resultado de la disminución del papel de las superpotencias en la región ${ }^{17}$.

\section{La interacción y la socialización dan lugar a una identidad colectiva.}

El realismo clásico se estructura a partir de tres supuestos fundamentales, a saber: el Estado-nación es el actor principal en las relaciones internacionales, hay una carencia de una autoridad central equivalente al gobierno nacional y la política internacional es esencialmente política de poder. El neorrealismo, por su parte, busca refinar el pensamiento realista clásico, asignándole mayor importancia al sistema internacional como estructura formada por Estados, aunque el poder sigue siendo una variable clave ${ }^{18}$. En otras palabras, lo que configura las relaciones políticas que tienen lugar entre sus miembros es la estructura y la política internacional es más que la suma de las políticas internacionales de los Estados y el equilibrio externo de otros agentes del sistema ${ }^{19}$. Por otra parte, los Esta- dos se comprometen en cálculos de costo-beneficio respecto de los cursos de acción alternativos de que dispone. En la medida en que estos beneficios previstos exceden a los costos, es probable que los Estados intenten hacer cambios en el sistema ${ }^{20}$.

Respecto de la región, los realistas establecen cuatro puntos fundamentales: predominio de las grandes potencias y dependencia de los Estados pequeños y débiles de las garantías de seguridad que ofrezcan los primeros; predominio de la lógica del equilibrio de poder, visualizando la estabilidad y la seguridad regionales en función de la preponderancia norteamericana; la competencia económica como causa potencial de conflictos regionales. Finalmente, son profundamente escépticos respecto de la capacidad de las instituciones regionales para manejar conflictos y preservar la paz ${ }^{21}$. En el ámbito regional, la visión "realista" y sus variantes ha predominado durante cincuenta años y ha puesto especial énfasis en la seguridad regional y particularmente en los conflictos de la Guerra Fría reflejados en la región, es decir, al accionar y la influencia de actores externos a la región. Desde la perspectiva realista, el interés por aspectos de la seguridad regional si-

${ }^{16}$ Un ejemplo de ello es: Buzan, Barry, "The Southeast Asian Security Complex", Contemporary Southeast Asia, $\mathrm{N}^{\mathrm{o}} 10$, pp. 1-15

${ }^{17}$ Roy, Denny, “Assessing he Asia-Pacific 'Power Vacuum,", Survival, Vol. 37, No 3, Autumn 1995, pp. 45-60.

${ }^{18}$ Dougherty, James E. y Pfaltzgraff, Robert, Teorías en Pugna de las Relaciones Internacionales, Buenos Aires, Grupo Editor Latinoamericano, 1993.

${ }^{19}$ Waltz, Keneth, Theory of International Politics, Reading Mass, Edison-Wesley Publishing Company, 1979.

${ }^{20}$ Gilpin, Robert, War and Change in World Politics, Nueva York, Cambridge University Press, 1981

${ }^{21}$ Acharya, Amitav, op. cit., 1999, pp. 2-4. 
gue siendo preponderante ${ }^{22}$, debido a la existencia de viejos conflictos dentro de la región, el surgimiento de nuevas amenazas externas (por ejemplo China), carreras armamentistas, etc., pero ha comenzado a evolucionar hacia un interés embrionario por los vínculos entre economía $\mathrm{y}$ seguridad regional ${ }^{23}$. Algunas visiones del realismo acerca del ARF aparecen como críticas, apuntando a que ofrecen poca ayuda a la seguridad regional e incluso que la socavan, principalmente en función de la contención de China ${ }^{24}$.

\section{Se presta creciente atención a} conceptos tales como la seguridad medioambiental.

Dentro de los nuevos enfoques que comienzan a aplicarse en la forma de visualizar la región se encuentra el constructivismo. Dicho enfoque plantea que no es posible hablar de la estructura del sistema internacional sin simultáneamente hablar acerca de la identidad e intereses de las unidades componentes ${ }^{25}$. En este sentido, este enfoque visualiza a los Estados como actores sociales, derivando su mirada desde el concepto sociológico de la acción, de acuerdo con el cual la conducta humana es regulada por reglas, normas, instituciones e identidades ${ }^{26}$. Este enfoque rechaza las visiones de realistas e institucionalistas en términos de que la cooperación depende necesariamente de una lógica de costo beneficio por parte de los Estados, y sostiene que la interacción creciente y la socialización redefinen sus intereses y desarrollan una identidad colectiva capaz de sobreponerse a la política de poder y al dilema de seguridad. La idea de una "comunidad de seguridad", planteada originalmente por Deutsch, ha sido rescatada por la perspectiva constructivista en función de una identidad colectiva (identidades múltiples), concebida en términos emergentes ${ }^{27}$ o condicionales mediante el desarrollo del principio de la autonomía y de un código de conducta regionales ${ }^{28}$, en que algunos procuran establecer elementos que reflejen esa identidad a partir de la utilización de una norma común o conjunto de ellas

${ }^{22}$ Dibb, Paul, "Towards New Balance of Power in Asia”, Adelphi, Paper 295, 1995

${ }^{23}$ Schultz II, Clifford J. y Ardrey IV, William J., "The Future Prospects of Sino-Vietnamese Relations: Are Trade and Commerce he Critical Factors for Sustainable Peace?", Contemporary Southeast Asia, Vol. 17, No 2, septiembre 1995, pp. 126-146. También ver Ackerman, Peter, “"The Economics Aspects of Pacific Security': A Reply”, Adelphi, Paper 275, marzo de 1993, pp. 31-35.

${ }^{24}$ Lim, Robin, "The ASEAN Regional Forum: Building on Sand", Contemporary Southeast Asia, Vol. 20, No 2, agosto, 1998, p. 115.

${ }^{25}$ Buzan, Barry y Little, Richard, International Sistems in World History. Remaking the Study of International relations, Nueva York, Oxford University Press, 2000, p. 42.

${ }^{26}$ Busse, Nikolas, "Constructivism and Southeast Asian Security", The Pacific Review, Vol. 12, No 1, 1999, pp. 39-60.

${ }^{27}$ Acharya, Amitav, "Ideas, Identity, and Institution Building: From the "ASEAN Way" to the AsiaPacific Way?", The Pacific Review, Vol. 10, N³, 1997, pp. 319-346.

${ }^{28}$ Nischalke, Tobias Ingo, "Insight from ASEAN's Foreign Policy Co-operation: The ASEAN Way, A Real Spirit or a Phantom”, Contemporary Southeast Asia, V. 22, № 1, abril 2000, pp. 89-112. 
(la "ASEAN way") para justificar determinadas políticas ${ }^{29}$.

\section{Se concibe el mundo como campo de interacción entre Estados soberanos en despiadada competencia.}

Con cierto predominio de la tradición realista, últimamente se observa un creciente interés académico por los conflictos intraestatales. Esto refleja la atención prestada al papel de la "diáspora" china en la región, debido a sus implicaciones para el área de la seguridad regional ${ }^{30}$. También se percibe en la creciente importancia del factor religioso ${ }^{31}$ vinculado al terrorismo (a partir del 11 de septiembre de 2001) y la preocupación respecto del sudeste asiático como la "periferia islámi- ca" ${ }^{2}$. Por otra parte, se rescata también al separatismo étnico como factor de inseguridad relevante ${ }^{33}$. A nivel interestatal, se intenta evaluar el impacto indirecto del sistema de Defensa de Misiles de Teatro (TMD) norteamericano en la región ${ }^{34}$, asociado a la competencia estratégica sinonorteamericana y se comienza a prestar también atención creciente a nuevas visiones de la seguridad tales como la seguridad medioambiental ${ }^{35}$. Las disputas limítrofes en el Mar del Sur de China, por su parte, mantienen su relevancia ${ }^{36}$, y desde un realismo bastante ortodoxo ${ }^{37}$, se percibe a China como una amenaza creciente, y se presta escasa atención a la perspectiva estratégica de esta última hacia el Pacífico, para contextualizar dichas disputas. Por otra parte, hay un interés creciente por las necesidades energéticas de China $^{38}$, aunque más asociadas a sus

${ }^{29}$ Busse, Nikolas, op. cit., 1999, pp. 46-48.

${ }^{30}$ Un ejemplo de ello en: Suryadinata, Leo, "Patterns of Chinese Political Participation in Four ASEAN States", Contemporary Southeast Asia, Vol. 15, № 3, diciembre 1993, pp. 292-308

${ }^{31}$ Ver May, R.J., "The Religious Factor in Three Movements: The Moro of the Philippines, the Malays of Thailand, and Indonesia's West Papuans", Contemporary Southeast Asia, Vol. 13, N 4, marzo 1992, pp. 396-414.

${ }^{32}$ Abusa, Zachary, “Tentacles of Terror: Al Qaeda's Southeast Asian Network”. Contemporary Southeast Asia, Vol. 24, No 3, diciembre 2002, pp. 427-465.

${ }^{33}$ Wee, Vivienne y Jayasuriya Kanishka, "New Geographies and temporalities of Power: Exploring the New Fault-lines of Southeast Asia", The Pacific Review, Vol. 15, Number 4, 2002, pp. 475-496.

${ }^{34}$ Tow, William T., “Asian perceptions of BMD: Defence or Desequilibrium?”, Contemporary Southeast Asia, Volume 23, $\mathrm{N}^{\circ}$ 3, diciembre 2001, pp. 379-400.

35 Ver Dupont, Alan, "The Environment and Security in Pacific Asia”, Adelphi Paper, $\mathrm{N}^{\circ}$ 319, International Institute for Strategic Studies, 1998. Ver también Rosemberg, David, "Environmental Pollution Around South China Sea: Developing a Regional Response", Contemporary Southeast Asia, Volume 21, Number 1, abril 1999, pp. 119-145.

${ }^{36}$ Ver Song, Yann-huei, "United States and territorial Disputes in the South China Sea", Maryland Series in Contemporary Asian Studies, N 1, 2002. También en Valencia, Mark, "China and the South China Disputes", Adelphi, Paper 298, 1995.

${ }^{37}$ Jae-Hyung, Lee, "China's Expanding Maritime Ambitions in the Western Pacific and the Indian Ocean", Contemporary Southeast Asia, Vol. 24, № 3, diciembre 2002, pp. 549-568.

${ }^{38}$ Andrews Speed, Philip, y Xuani Liao, "The Strategic Implications of China's Energy Needs, Adelphi Paper, $\mathrm{N}^{\circ}$ 346, The International Institute for Strategic Studies, 2002. 
reforzados vínculos con Eurasia que a su impacto en el Mar del Sur de China.

\section{La particularidad del realismo chino es el énfasis en el desarrollo económico.}

La percepción de China como un factor de inestabilidad se sustenta también en la visión teórica preponderante en China respecto del sistema internacional, reflejando la preeminencia del paradigma realista, de acuerdo con el cual el mundo es casi exclusivamente un campo de interacción entre Estados soberanos vinculados a través de una despiadada competencia. A pesar de reconocer la interdependencia y el multilateralismo, no parecerían interesados en la forma en que esas nuevas fuerzas reformulan las estructuras y procesos del sistema internacional. Más bien, conciben el crecimiento de las redes transnacionales y multilaterales a través de un prisma estado-céntrico, que solo apunte a evaluar cómo China podría sacar ventaja de ese nuevo entorno externo para proteger y maximizar su interés nacional. Este es el motivo primario y directo, y el resto de las dinámicas son secundarias y permean dicho interés nacional. La particularidad del realismo chino es el énfasis que asignan al desarrollo económico y tecnológico. Dicha particularidad es atribuible a la reciente convicción china de que actualmente la política internacional se caracteriza por la búsqueda de "poder comprehensivo" sobre una amplia gama de campos de batalla en las áreas económica, política y tecnológica. Desde la perspectiva china, las diferencias ideológicas son ahora menos importantes y los intereses nacionales, especialmente los económicos, han incrementado su importancia ${ }^{39}$.

\section{LA PERCEPCIÓN CHINA RESPECTO \\ DE LOS ORGANISMOS \\ MULTILATERALES Y EL ARF.}

Los especialistas chinos consideran inevitable el incremento del multilateralismo y ven que el involucramiento selectivo en dichos esquemas es menos riesgoso que su no participación. La orientación china hacia la solución bilateral de las controversias y hacia el equilibrio de poder con las potencias externas han dado un bajo perfil a esta participación, pero las condiciones actuales la inducen a un mayor involucramiento. Además, su objetivo de construir un mundo multipolar pasa por contar con estructuras multilaterales que contrapesen la fuerte presencia norteamericana en un mundo unipolar. Algunos ejemplos claros son la participación de China en la Organización de Cooperación de Shanghai, surgida del "Grupo de Shanghai" y que integran China, la Federación de Rusia y la mayoría de los países del Asia central (excluyendo Turkmenistán); El Foro Regional de la ASEAN también se transforma en un espacio de oportunidades que permiten compensar, de alguna manera, el papel regional de Estados Unidos, aspecto refor-

\footnotetext{
${ }^{39}$ Yong, Deng, op. cit., pp. 308-329.
} 
zado por la percepción heterogénea respecto de China por sus miembros.

\section{Ha variado la perspectiva china sobre los esquemas de seguridad multilateral regional.}

China a variado su perspectiva respecto de los esquemas de seguridad multilateral regional. En esta materia la política China ha sido vaga y cautelosa, aunque ha mostrado su buena voluntad para participar en ellos y siempre ha tenido presente la actitud de otros actores en la zona. China basa esta participación en que los países deben ser tratados como iguales en virtud de los principios de la coexistencia pacífica, es decir, ningún país debe buscar hegemonía, o esferas de influencia, organizar o unir algún bloque militar dirigido contra otros países; ningún país debe intervenir en los asuntos internos de otro; el desarme y control de armas debe llevarse a cabo de manera posible y razonable; hay que esforzarse por finalizar las carreras armamentistas y prevenir la proliferación nuclear; y las disputas territoriales, fronterizas y otras diferencias entre Estados regionales deben solucionarse pacíficamente, sin recurrir a la fuerza.

\section{LA RELACión ChINA-ASEAN}

El respaldo chino al proceso de pacificación de Camboya contribuyó a generar los espacios para mejorar las relaciones con los países de Indochina y de la $\mathrm{ASEAN}^{40}$, que se habían deteriorado a raíz del problema de las islas Spratly (Nansha). El primer país que propició un acercamiento con China fue Tailandia, cuyo comercio con ella había aumentado desde la década de los ochenta. El primer paso efectivo se dio por la amenaza vietnamita mediante la venta de pertrechos militares a Tailandia, el apoyo de Tailandia al Khmer Rouge de Camboya, y presión de la presencia de fuertes unidades chinas en la frontera, que limitaba la posibilidad de un ataque vietnamita a territorio tailandés, lo que estrechó los lazos militares. Además, China había cortado el apoyo a los comunistas tailandeses ${ }^{41}$.

El paso siguiente fue el mejoramiento de las relaciones con Singapur e Indonesia, ya que ambos países estaban preocupados por la presencia soviética en la zona. En el primer caso se restablecieron los lazos diplomáticos, mientras que en el segundo las relaciones se habían deteriorado desde 1965, por el supuesto apoyo chino al golpe de Estado propiciado por los comunistas indonesios ${ }^{42}$, que derivó en un congelamiento de las relaciones en octubre de $1967^{43}$. Esto también reflejaba la difícil situación de la minoría

\footnotetext{
${ }^{40}$ Soled, Debra E., China, A Nation in Transition, op. cit., p. 219.

${ }^{41}$ Ibid., p. 221.

${ }^{42}$ Ibid.

${ }^{43}$ Sukma, Rizal, "Recent Developments in Sino-Indonesian Relatións: An Indonesian View", Contemporary Southeast Asia, Vol. 16, № 1, junio 1994, p. 37.
} 
étnica china en dicho país, que persiste en la actualidad ${ }^{44}$. Cabe observar que las relaciones de China e Indonesia se reanudaron en $1990^{45}$. Con Malasia, China utilizó la misma política de cortar el apoyo al Partido Comunista Malayo, lo que obedecía también a la necesidad china de contar con el respaldo de los chinos de ultramar, malayos, a su propio proceso de desarrollo ${ }^{46}$.

\section{Persiste la difícil situación de la minoría étnica china.}

La relación entre la ASEAN y China comienza a tener rasgos definidos a partir del 19 de julio de 1991, cuando el Ministro de Relaciones Exteriores chino Qian Qichen, asistió a la inauguración de la vigésimo cuarta reunión de la AMM en Kuala Lumpur, invitado por el gobierno malayo. En dicha reunión, China expresó su interés por fortalecer la cooperación con la ASEAN, particularmente en materia de ciencia y tecnología. Ante la respuesta positiva de la ASEAN, se buscó establecer lazos de carácter más formal.

La ASEAN estaba procurando que la disputa sobre las Spratly (Nansha) no obstaculizara sus propósitos de crear una Zona de Paz, Libertad y Neutralidad en el sudeste asiático y el desarrollo econó- mico que ésta acarreara. El principal problema que enfrenta la ASEAN es el de mantener una posición conjunta respecto de China, sobre lo cual no todos sus miembros están de acuerdo. Cabe notar que la ASEAN toma sus decisiones por consenso; además, los tres reclamantes de la controversia que pertenecía a la ASEAN no tienen ninguna inclinación a comprometerse entre ellos por problemas de soberanía. Aunque Vietnam ha sido aceptado como miembro, es probable que el resto de estos países trate de evitar ser llevados a la confrontación de Vietnam con China. Hay que destacar que Tailandia y Singapur no tienen la calidad de Estados reclamantes ${ }^{47}$.

A pesar de los buenos augurios del acercamiento con ASEAN, un hecho enrareció las relaciones en 1992, y fue la incorporación a la legislación china de la autorización para la acción militar contra el tráfico marítimo que violara la soberanía china ${ }^{48}$. Esto produjo la reacción de la ASEAN con la "Declaración sobre el Mar del Sur de China"49, aprobada el 22 de julio de 1992 en Manila, Filipinas, que constituyó un hito en la manera en que los países de la ASEAN abordaban sus disputas con China. Dicho documento apuntaba a enfatizar la necesidad de resolver todos los problemas de soberanía y jurisdiccionales respecto del Mar del

${ }^{44}$ Ibid., p. 43.

${ }^{45}$ Drake, Earl, "Indonesia and China: Old Habits and New Internationalism", The Pacific Review, Vol. 4, No 3, 1991, p. 218

${ }^{46}$ Soled, Debra E., China, A Nation in Transition, op. cit., p. 221.

${ }^{47}$ Valencia, Mark, "China and the South China Disputes", Adelphi, Paper 298, 1995, pp. 42-49.

${ }^{48}$ Haas, Michael, “Asean's Pivotal Role in Asia-Pacific Regional Cooperation”, Global Governance, Vol. 3, No 3, sept-dic., 1997, p. 340.

${ }^{49}$ Basado en el texto original de la página oficial de ASEAN en Internet $<\mathrm{http}$ ://www.aseansec.org $>$. 
Sur de China por medios pacíficos, establecer un código de conducta sobre el particular y explorar las posibilidades de cooperación y desarrollo económico.

\section{La ASEAN procuró incorporar a China en temas de interés común.}

La ASEAN procuró incorporar a China en múltiples temas de interés común, incluida la discusión sobre el problema de las Spratlys (Nansha). Como consecuencia de ello, una delegación de la ASEAN visitó Beijing entre el 11 y el 18 de septiembre de 1993, se emitió una declaración conjunta el 13-14 de septiembre de 1993 en que se acordaba reunirse para explorar la posibilidad de establecer una relación de carácter consultivo con China. Ambas partes sostuvieron conversaciones exploratorias que buscaban fortalecer la cooperación, lo que se tradujo en la creación de dos comités conjuntos, uno sobre cooperación en economía y comercio y el segundo sobre cooperación en ciencia y tecnología.

El intercambio de documentos entre el ministro de relaciones exteriores chino y el Secretario General de la ASEAN, el 23 de julio de 1994, formalizó la creación de los comités conjuntos. Además, en esa reunión se acordó realizar consultas sobre problemas de interés mutuo en materia de seguridad y políticas entre fun- cionarios de alto nivel. Sus resultados inmediatos fueron la aceptación y la discusión por parte de China del problema de las Spratlys (Nansha), sobre la base de la Convención de las Naciones Unidas sobre el Derecho del $\mathrm{Mar}^{50}$.

La figura jurídica que le permite a la ASEAN mantener un diálogo institucional sobre temas de su interés con países que no son miembros de la Asociación es la de "socio de diálogo", en virtud de la cual China se incorporó en 1996. La figura se creó en 1977 y los primeros países en obtener ese estatus fueron Australia, Japón y Nueva Zelandia. Posteriormente lo adquirieron Estados Unidos, Canadá, la Unión Europea y Corea del Sur. Finalmente se agregaron Rusia, China y la India. En julio de 1996, China acordó incorporarse con carácter de socio de Diálogo Pleno en la vigésimo novena reunión de ministros de relaciones exteriores de ASEAN realizada en Yakarta, a la que asistió el ministro de relaciones exteriores de China por primera vez en este carácter. En el plano de la seguridad, China se unió al Foro Regional de la ASEAN en $1994^{51}$.

Las relaciones de China con la ASEAN pasan por un buen momento. Dicho mejoramiento se reflejó en un aumento del comercio, que el primer semestre de 2003 alcanzó a US\$23.550.000, lo que significa un incremento del $18,7 \%$ respecto del año anterior ${ }^{52}$. Un hito im-

\footnotetext{
${ }^{50}$ Haas, Michael, “Asean's Pivotal Role in Asia-Pacific Regional Cooperation”, op. cit., p. 343.

${ }^{51}$ Cheng, Joseph Y. S., "Sino-ASEAN Relations in the Early Twenty-first Century", Contemporary Southeast Asia, Vol. 23, No 3, diciembre 2001, p. 421.

52 "Fair indicates great trade potential between China, ASEAN", (2003-08-27 14:51), <http:// www1.chinadaily.com.cn/en/doc/2003-08/27/content_258844.htm>.
} 
portante que refleja el estado de las relaciones es el acuerdo de noviembre de 2002 para constituir un Área de Libre Comercio en un proceso que finalizará el 2015, y en 2010 en el caso de las economías más desarrolladas. Otro acuerdo suscrito en 2002 apunta a la adopción de un código de conducta respecto de las disputas en el Mar del Sur de China. Ambos esfuerzos reflejan, por una parte, el deseo de ambos de minimizar las tensiones diplomáticas y ampliar las oportunidades económicas y, por la otra, responder a la eficacia norteamericana mejorando los lazos regionales sobre la base de la lucha contra el terrorismo ${ }^{53}$. Sin embargo, también persisten los esfuerzos por mantener negociaciones en el plano bilateral respecto de la disputa del Mar del sur de China $^{54}$.

China, Estados Unidos y los equiliBRIOS ESTRATÉGICOS REGIONALES.

A raíz del pragmatismo que se dio, en el período de reforma chino la importancia de la política se desplazó hacia la economía como factor determinante de la política exterior. La necesidad de estabilidad interna, propicia para maximizar el crecimiento, acentuó la búsqueda de una política exterior que garantizara paz y seguridad, de tal modo que el país pudiese orientar todas sus energías hacia la modernización económica. Cabe señalar que en este período perdió vigencia el concepto de triángulo estratégico, es decir, la relación triangular que contraponía los intereses chinos "imperialismo" norteamericano y al "hegemonismo" soviético. Luego del distanciamiento sinonorteamericano, el acercamiento sinorruso flexibilizó dicho enfoque, hasta que finalmente se disolvió al desaparecer la Unión Soviética, debiendo China reorientar sus opciones estratégicas. Ésta se centró en el Asia-Pacífico, y apuntó también a construir un sistema multipolar que contrapesara el accionar norteamericano.

\section{El pragmatismo desplazó la importancia de la política hacia la economía.}

En la actualidad, el escenario del este de Asia presenta cierta dualidad. Por un lado, se ha incrementado la percepción de amenaza en el noreste asiático debido al despliegue en Asia oriental del Sistema de Defensa de Misiles de Teatro (TMD) como parte del nuevo Sistema de Defensa de Misiles Norteamericano, la potencial declaración de independencia de Taiwán, el potencial rearme de Japón y el incremento de las tensiones en la península de Corea. En dichos ámbitos, Estados Unidos, rival estratégico de China, posee la capacidad de iniciativa estratégica y de construcción de escenarios favorables.

${ }^{53}$ Strategic Survey, The International Institute for Strategic Studies, London, Oxford University Press, 2003; pp. 252-253.

${ }^{54}$ Storey, Adam James, "China, the Philipines and the South China Sea Dispute", Contemporary Southeast Asia, Vol. 21, No 1, abril 1999, p. 96. 
La relación sinonorteamericana pasa por un buen momento debido al impacto de los atentados del 11 de septiembre, aunque dista mucho de ser óptima. Esta relación, con sus complejidades, con períodos de distensión y conflicto, ha marcado el período de la reforma y ha reconfigurado los diversos contextos regionales. El factor que acrecienta la sensibilidad china hacia dicha relación posiblemente sea el peso del nacionalismo en la política exterior china, que ha generado rigideces que limitan en gran medida su margen de maniobra. Un ejemplo claro de esta sensibilidad es el conflicto con Taiwán y la crisis que podría producirse si ésta dictara la independencia. Ello se explica porque el proyecto de unificación nacional es un elemento fundamental de la estrategia de legitimación del régimen dirigido por el Partido Comunista Chino durante el período de la reforma iniciada por Deng Xiaoping. Bajo este prisma, pueden incorporarse también los derechos reclamados por China sobre la mayor parte del Mar del Sur de China invocando argumentos históricos, tema que la enfrenta a numerosos países miembros de la ASEAN así como la disputa sobre las islas Senkakus, con Japón.

La percepción norteamericana de un incremento del poder militar chino y el interés por proteger las líneas marítimas que cruzan el estrecho de Malaca han hecho que, además de sus bases en el noreste asiático, Estados Unidos busque mejores bases y facilidades en Filipinas,
Singapur y otros lugares del sudeste asiático no necesariamente permanentes pero que al menos le permitirán un rápido despliegue en la región ${ }^{55}$. Este potencial "retorno" norteamericano a la región obedecería en la práctica a una estrategia de contención de largo plazo respecto de China, que pese a tener indicadores más claros en el noreste asiático, principalmente en el conflicto de Taiwán, no excluye la posibilidad de un incremento de las tensiones respecto de la disputa de las islas Spratly.

\section{Pese a pasar por un buen momento, la relación sinonorteamericana no es óptima.}

La postura norteamericana dista de ser neutral, pero no es en ningún caso agresiva. Sin embargo, lo delicado de dicha disputa, enmarcada en la competencia por recursos energéticos y principalmente por la protección de las rutas de transporte de dichos recursos a través del Mar del Sur de China, la tornan en un tema extremadamente sensible. Esto se debe a la impresión que se tiene sobre la transición en la perspectiva estratégica china, que incluye su capacidad militar y en la forma en que puede utilizarse en la región.

La potencial independencia de Taiwán y probable involucramiento militar norteamericano, se constituye en el peor escenario visualizado por las autoridades chinas, en el sentido de las dificul-

${ }^{55}$ Tangredi, Sam J., Globalization and Marítime Power, Washigton D.C., National Defense University Press, 2002 p. 298. 
tades que plantea un conflicto militar con ese país. Las consecuencias de una crisis con Taiwán no se limitarían al noreste asiático sino que se proyectarían al Asia sudoriental, ya que ante la imposibilidad de ocupar Taiwán la alternativa podría ser ocupar las islas actualmente ocupadas por Taiwán en las islas Spratly, en el Mar del Sur de China. China ha hecho grandes esfuerzos para enfrentar esta clara asimetría desfavorable. Los estrategas chinos creen identificar una serie de debilidades militares y políticas norteamericanas (elevada dependencia de sistemas de armas y tecnología altamente sofisticada, aversión cultural a enfrentar un gran número de bajas, etc.).

\section{Aunque dista de ser neutral, la posición norteamericana} no es agresiva.

El eje de la evaluación china de la perspectiva estratégica norteamericana dista mucho de ser favorable, ya que considera que el interés norteamericano por mantener su posición dominante en el mundo pasa por impedir que un Estado o grupo de Estados de eurasia, amenace su posición e intereses, para así mantener su dominio sobre la norma y regulaciones internacionales, y las relaciones internacionales y poder introducir el estilo anglosajón de democracia. En este sentido, Estados Unidos procura mantener su posición de liderazgo en los campos econó- mico y tecnológico, intentando dominar los recursos estratégicos y la reglamentación económica, además del predominio militar sobre sus rivales ${ }^{56}$.

\section{Cambios en la Doctrina Estratégica China}

Los cambios fundamentales experimentados por la doctrina estratégica china han puesto acento en la forma de abordar la inestabilidad regional y los conflictos territoriales en la periferia sudoriental y sudoccidental, otorgando al Mar del Sur de China y el Estrecho de Formosa una importancia creciente, sin abandonar la perspectiva de un conflicto nuclear en el futuro. Para ello, China desarrolló una estrategia de defensa nacional orientada a aumentar su capacidad nuclear y mejorar el poder de fuego convencional del Ejército Popular de Liberación (EPL).

En el período comprendido entre mediados de los años ochenta y el decenio de 1990, comenzó la transición hacia una fuerza militar más moderna y flexible, orientada a centrar la atención sobre un conflicto militar inesperado e intenso a lo largo de las fronteras y territorios marítimos. Uno de los cambios principales de la doctrina estratégica china esbozados a comienzos de la reforma fue la transición de la "guerra popular prolongada" hacia una "guerra popular en condiciones modernas". Este cambio significó esencialmente el tránsito de una guerra de gue-

${ }^{56}$ Jin, Canrong, "Uncertaineties caused by violation of tradition", Contemporary International Relations, China Institute of Contemporary International Relations, Vol. 13, N ${ }^{\circ}$, septiembre 2003, en p. 52. 
rrillas a un concepto que incorporaba la guerra posicional, el uso de armamento moderno y de fuerzas combinadas, y a partir de la base de que había que defender centros urbanos importantes y de que un ataque nuclear a su territorio era inevitable, lo que cambió la noción de disuasión por la de represalia. En este nuevo escenario, una posible guerra ya no se daría en el interior de China sino más bien en sus fronteras.

A mediados de los años ochenta se produjo un segundo cambio de política que acentuaba la posibilidad de una guerra local en la periferia del país. Esta guerra tendría un carácter más político que una guerra mayor, sería difícil distinguir entre las victorias y las derrotas y se finalizaría por vías diplomáticas, con objetivos limitados, buscando apoyar iniciativas diplomáticas, intimidar la psicología del enemigo y adquirir recursos económicos. La guerra local tiende a no ser nuclear, orientada hacia la alta tecnología y se desarrolla en un espacio y tiempo limitados, pudiendo comprometer a tropas, lo que permite gran movilidad y sorpresa y conducir a una solución rápida. Luego de la Guerra del Golfo se le agregó el concepto de "guerra limitada en condiciones de alta tecnología", que validó la idea de una guerra limitada y aceleró el proceso de modernización en términos tecnológicos y organizacionales, ya que el conflicto ponía de manifiesto la debilidad china ante el despliegue de la nueva tecnología militar norteamericana.
Un cambio doctrinario con claras consecuencias en el sudeste asiático se dio en la elaboración de una estrategia marítima china hacia el Pacífico ${ }^{57}$. Pese a que históricamente China ha sido un poder continental más que marítimo, el desarrollo de una estrategia de desarrollo económico que ha privilegiado el desarrollo de zonas económicas especiales a lo largo de las costas chinas desde fines de los años setenta cambió la "sensibilidad" china respecto del mar, ya que alrededor del $50 \%$ de su economía depende del comercio exterior y de éste, el $90 \%$ es transportado por vía marítima. La necesidad de implementar una nueva estrategia también obedeció a necesidades presupuestarias de la Armada. En este sentido, la misión de ésta cambió del tradicional papel de defensa costera a una "defensa activa costa afuera".

\section{Un cambio de política aumentó la posibilidad de una guerra en la periferia del país.}

La Armada del EPL delineó tres áreas estratégicas marítimas que debería ser capaz de controlar en distintas etapas. La primera de ellas incluía el mar Amarillo, frente a Corea y Japón, el oeste del Mar de China oriental incluyendo Taiwán y el Mar del Sur de China. Esta área era considerada vital para los intereses nacionales (reclamaciones territoriales, recursos naturales y defensa costera). Era denomi-

${ }^{57} \mathrm{El}$ arquitecto de la actual estrategia naval china fue el almirante Liu Huaqing, comandante de la Armada del EPL de 1982 a 1987 y luego Vice-jefe de la Comisión Militar Central desde 1989 a 1997, cargo de enorme influencia. 
nada "primera cadena de islas" e incluía una línea que recorría de norte a sur desde las Aleucianas, y atravesaba las Kuriles, Japón, las Ryuku, Taiwán, las Filipinas e Indonesia. La idea era controlar esa zona alrededor del año 2000. La segunda área marítima, denominada "segunda cadena de islas", corría desde las islas Kuriles, a través de Japón, las Bonin, las Marianas y las Carolinas y permitía controlar vastas áreas oceánicas. Esta segunda etapa debería completarse alrededor del año 2020. La tercera etapa de la estrategia marítima de Liu describe a la Armada del EPL como fuerza global o, en el peor de los casos, capaz de actuar en el Pacífico para el $2050^{58}$.

\section{Las principales amenazas externas siguen proviniendo del mar.}

El cambio de doctrina en el ámbito naval apuntaba a darle un papel preponderante a la proyección marítima china. Por otra parte, desde la perspectiva china, las principales amenazas externas siguen proviniendo del mar. Si bien las necesidades coyunturales son impedir la independencia de Taiwán y prevenir amenazas en su periferia, el claro objetivo de la estrategia marítima china es negar el uso del mar a los norteamericanos y sus aliados en Asia y en Japón, así como respaldar sus pretensiones en las disputas limítrofes con los países de la región.

Si bien China ha incrementado su presupuesto militar, la modernización militar sigue siendo la cuarta prioridad de su proceso de modernizacón y no hay indicios que nos hagan pensar que estas opciones políticas hayan cambiado, o que quiera entrar en una carrera armamentista con Estados Unidos. Lo que en efecto parece ser claro, es que China quiere alejar a Estados Unidos de sus costas. Esto se tradujo en que debió enfrentar un proceso de desarrollo y adquisición de armamento que tropezó con una serie de dificultades. Debido a obstáculos estructurales es que la incorporación de armamento de alta tecnología debía ser gradual, pues las limitaciones administrativas, tecnológicas y presupuestarias obligaron a orientar los esfuerzos hacia la selección de armamento muy avanzado pero de volumen limitado ${ }^{59}$.

En el sudeste asiático, los cambios de doctrina estratégica y el consiguiente proceso de modernización militar chino se han dado en el contexto de una disminución significativa del papel desempeñado por Rusia y Estados Unidos en la región, generando un "vacío de poder". Como consecuencia de ello, se ha produ-

${ }^{58}$ Un concepto clásico en estrategia marítima es el "dominio del mar", consistente en la capacidad de usar el mar, al mismo tiempo que negarlo a un adversario. El concepto de "control del mar" se refiere a la capacidad de dominar un área oceánica discreta por un período limitado, suficiente para alcanzar metas estratégicas limitadas. El contrapunto del "control del mar" es el de "negación del mar", es decir, negar a un adversario el uso de una discreta área marítima, sin usarla uno mismo. Dentro de la perspectiva del Almirante Liu, no queda claro si las tres distintas etapas estaban definidas en términos de control o negación del mar, ya que solamente se plantean en términos de áreas de interés estratégico.

${ }^{59}$ Cabe señalar que parte importante del equipamiento convencional del EPL es anticuado y sus diseños datan principalmente de los sesenta y setenta, no así su armamento nuclear. 
cido una redefinición de los sistemas de alianzas en la región, en función de la potencial amenaza china, lo que ha tenido como principal consecuencia la incorporación de los países de Indochina a la ASEAN. Esta incorporación tiene la obvia consecuencia de aumentar aún más la heterogeneidad de la organización, además de incorporar a países con ostensible retraso económico respecto de los miembros originales. Pero, por otra parte, se da el trasfondo de la necesidad de aumentar la seguridad regional ante un posible incremento de los conflictos con China en el caso de Vietnam y, de Myanmar, a contrapesar la creciente influencia china. Cabe destacar que el principal interés estratégico chino en Myanmar es conseguir el paso hacia la bahía de Bengala y el mar de Andamán a través de la frontera sudoccidental de China y además impedir que India consiga dominar el Océano Indico.

La heterogeneidad de la ASEAN dificulta una apreciación de seguridad común respecto de China, a lo que contribuyen las disputas territoriales dentro de los países miembros. A esto debemos sumar el hecho de que el conflicto de las Spratly, que involucra a múltiples actores, tiene un alto potencial desestabilizante, debido a los recursos energéticos que posee y a su importancia estratégica. Cabe notar que Vietnam mantiene disputas te- rritoriales con China pero ya no cuenta con el apoyo militar que le proporciona la Unión Soviética, lo que torna a China en una amenaza más inminente.

\section{Los sistemas regionales de alianza se han redefinido en función de la potencial amenaza china.}

Por otra parte, es indudable que el mar del Sur de China no constituye la principal prioridad estratégica china, que está más focalizada en el noreste asiático, y permite un rango de flexibilidad mayor ${ }^{60}$. El problema de la competencia estratégica por recursos energéticos y por el control de las rutas marítimas que cruzan el mar del Sur de China se superpone a los problemas de soberanía. Sin embargo, la competencia estratégica con Estados Unidos posee mayor relevancia a la hora de explicar los factores que articulan la lógica de cooperación y conflicto de China en la región.

\section{LA SEguridad ENERGÉTICA DE CHINA Y} SUS CONSECUENCIAS PARA EL SUDESTE

$$
\text { ASIÁTICO }
$$

La seguridad energética, es decir, garantizar la permanente disponibilidad de variadas formas de energía, en suficientes cantidades y a precios razonables ${ }^{61}$, ha

${ }^{60}$ Shee Poon Kim, "The South China Sea in the China's Strategic Thinking”, Contemporary Southeast Asia, Vol. 19, № 4, marzo 1998, p. 385

${ }^{61}$ Khatib, Hisham, "Energy Security”, Capítulo IV; en Goldemberg, José (ed.), World Energy Assessment: Energy and the Challenge of Sustainability, Programa de las Naciones Unidad para el Desarrollo (PNUD), Departamento de Asuntos Económicos y Sociales (UNDESA) and World Energy Council (WEC). p.112. 
incrementado su prioridad en las necesidades estratégicas chinas. El rápido crecimiento económico de China, registrado en el período de la reforma, lo ha convertido de autosuficiente a importador neto de recursos energéticos. Actualmente, el país es el segundo consumidor del mundo, después de Estados Unidos, lo que ha alterado el interés por ciertas áreas de la periferia china, que poseen dichos recursos. Ejemplos de ello son el creciente interés por los recursos energéticos que podrían contener las islas Nansha (Spratly) o los recursos provenientes en tránsito desde el Asia central.

\section{La seguridad energética ha aumentado las prioridades de las necesidades estratégicas.}

La perspectiva china en materia de seguridad energética apunta principalmente a maximizar la producción nacional de gas y petróleo, invertir en la producción de ultramar y aumentar los vínculos con los países exportadores. Para ello, se le está dando un enfoque estratégico en un sentido amplio, pero relegando a un lugar muy secundario medidas económicas tales como la liberalización del mercado interno, respecto de la disminución de la demanda. Por otra parte, recién comienzan a implementarse medi- das de respuesta de emergencia ${ }^{62}$. La creciente importancia de los suministros energéticos transportados desde países del Medio Oriente (entre ellos Omán, Yemen e Irán) hacia China, ha aumentado la importancia de las líneas de comunicación marítima que cruzan los estrechos del sudeste asiático hacia el Mar del Sur de China, dando lugar a que Estados Unidos se perciba como la principal amenaza a su seguridad energética ${ }^{63}$ y haciendo priorizar los vínculos energéticos continentales (Rusia, Kazajstán, etc.) ${ }^{64}$.

La vinculación energética china con el sudeste asiático se ha incrementado también en términos positivos. Muestra de ello es la intensificación de los esfuerzos de los países de la ASEAN por diversificar sus fuentes energéticas y cooperar con China (además de Japón y Corea del Sur) para garantizar la cooperación energética regional. En dicha cooperación, el tema de la seguridad energética se ha hecho cada vez más relevante ${ }^{65}$. Cabe recordar también que Indonesia es uno de los proveedores importantes de recursos energéticos para China en el sudeste asiático.

\section{Conclusiones}

La relación de competencia estratégica sinonorteamericana es un elemento sustantivo para la seguridad de Asia orien-

\footnotetext{
${ }^{62}$ Andrews-Speed, Philip, Xuanli Liao y Dannreuther, Roland, op. cit., pp. 42-43.

${ }^{63}$ Strecker Downs, Erica, "China's Quest for Energy Security”, Publicación electrónica de RAND Corporation, 2000, p. 44, <http://www.rand.org/publications/MR/MR1244/>.

${ }^{64}$ Ibidem, pp. 45-46.

${ }^{65}$ Agence France Presse, 4 de julio de 2003, “ASEAN to Work with China, Japan, South Korea for Energy Security".
} 
tal. Pese a que el tema del terrorismo ha generado expectativas de cooperación en la relación entre ambos, ello no ha modificado sustancialmente las opciones estratégicas de ninguno de ellos. Esto se debe en gran medida, a la impresión negativa de China respecto de la fuerte influencia norteamericana en la región. Esta competencia se refleja en múltiples áreas, tales como el regreso norteamericano al sudeste asiático, el debate respecto del Sistema de Defensa de Misiles de Teatro (TMD) o los lazos de seguridad norteamericanos con Taiwán, con el agravante de una eventual independencia de la isla. En este sentido, las disputas en el mar del Sur de China, que involucran a China y varios países del sudeste asiático, serían un espacio sutil de competencia hegemónica sinonorteamericana, a largo plazo, la opción estratégica china en dicha zona parece más orientada a reforzar su presencia en la zona que a gatillar una crisis importante o crisis parciales.

\section{La relación de competencia sinonorteamericana es un elemento sustantivo para la seguridad de Asia oriental.}

Por su parte, Estados Unidos ha comenzado a distanciarse gradualmente desde un papel neutral en las disputas del Mar del Sur de China, y ha incrementar su presencia militar en el ámbito regional ${ }^{66}$. Este país declara su interés en la solución pacífica de los conflictos señalados, no involucrándose abiertamente en ellos, basándose en su intención de garantizar la libertad de navegación en la región.

Las visiones optimistas que ven el papel norteamericano como estabilizador de las líneas marítimas de la región ${ }^{67}$, no tienen en cuenta la amenaza que esto podría representar a largo plazo para China. Las crecientes necesidades energéticas del país, que lo han convertido desde un país autosuficiente en materia energética en importador neto de estos recursos, han incrementado la importancia de contextos regionales tales como el mar del Sur de China y el Asia central, desarrollando en el primero un mayor grado de asertividad con el fin de obtener o garantizar el flujo energético para su desarrollo económico. En el sentido amplio, el interés estratégico militar dirigido hacia cómo abordar las inestabilidades regionales y disputas territoriales en la periferia sudoriental, que ha otorgado importancia creciente al mar del Sur de China, y sudoccidental está estrechamente relacionado con la seguridad energética de China. Si a ello sumamos el peso del nacionalismo en la política exterior china, vemos que sus pretensiones de soberanía en el área no son susceptibles de una negociación flexible.

A partir de lo anterior, se ha modificado el criterio de China respecto de los esquemas de seguridad multilateral regio-

${ }^{66} \mathrm{Wu}$ Ximbo, "U.S. Security Policy in Asia", Contemporary Southeast Asia, Vol. 22, No 3, diciembre 2000 , p. 486.

${ }^{67}$ Feigenbaum, Eva A., "China's Military Posture and the New Economic Geopolitics", Survival, Summer, 1999, Vol. 41, № 2, pp. 79-81. 
nal. China está abandonando su política aparentemente vaga y cautelosa respecto de los esquemas de seguridad multilateral, y buscando una mayor participación. Esto se debe fundamentalmente a que su objetivo de construir un mundo multipolar pasa por contar con estructuras multilaterales que contrapesen la fuerte presencia norteamericana en el este de Asia (concentrada principalmente en el noreste). En este sentido, una relación positiva con los países de la ASEAN y la incorporación en sus instancias de seguridad apuntan a construir una estabilidad amplia que contrapese la reconstitución de la presencia norteamericana en el sudeste asiático, algo debilitada durante la post Guerra Fría. El Foro Regional de la ASEAN, por su flexibilidad, por el bajo grado de formalización y por la percepción heterogénea de los distintos actores respecto de China, se presenta como una instancia oportuna para garantizar una base de estabilidad en la situación estratégica regional, abriendo además espacios más amplios de negociación, pero sin discutir directamente de temas de soberanía que se mantienen en el plano bilateral. Al respecto, una mayor formalización de esta instancia podría disminuir su relevancia.

\section{Las pretensiones de soberanía no son susceptibles de una negociación flexible.}

El incremento de la competencia estratégica entre Estados Unidos y China ha aumentado la importancia del Foro Regional de la ASEAN (ARF) como base de la arquitectura de la seguridad regional. Sin embargo, a pesar de un mayor grado de integración económica regional y de que el problema del terrorismo ha facilitado la construcción de consensos al interior del ARF, las disputas limítrofes en el Mar del Sur de China, vinculadas los recursos energéticos que posee, continúan siendo un factor de inseguridad clave que amenaza la estabilidad regional y dificulta el desarrollo de dicho organismo. 